06

\title{
Генерация суммарной частоты от тонкого сферического слоя. II. Анализ решения
}

\author{
(C) А.А. Шамына, В.Н. Капшай
}

Гомельский государственный университет им. Ф. Скорины, 246019 Гомель, Беларусь

e-mail: anton.shamyna@gmail.com, kapshai@rambler.ru

Поступила в редакцию 23.02.2018 г.

Проанализировано влияние угла между волновыми векторами падающих волн (угла раскрытия) и типа анизотропии нелинейного слоя на форму диаграммы направленности гармоники суммарной частоты, генерируемой двумя плоскими электромагнитными эллиптически поляризованными волнами от тонкого сферического оптически нелинейного слоя, нанесенного на поверхность диэлектрической сферической частицы, помещенной в диэлектрик. Анализ показал, что для малого радиуса тонкого сферического нелинейного слоя лишь при некоторых типах анизотропии вид диаграммы направленности существенно меняется с увеличением угла раскрытия: главные лепестки смещаются к направлению, обратному сумме волновых векторов падающих волн. Для трех типов анизотропии диаграммы направленности имеют близкую форму. Также установлено, что для одного из типов анизотропии форма диаграммы направленности не меняется при изменении угла раскрытия. Выявлены математические свойства функций, описывающих пространственное распределение генерируемой гармоники. В частности, обнаружено, что при падении линейно поляризованных волн на тонкий нелинейный сферический слой, обладающий исключительно киральными или исключительно некиральными нелинейными свойствами, генерируется линейно поляризованное излучение гармоники суммарной частоты.

DOI: $10.21883 /$ OS.2018.07.46269.56-17

\section{Введение}

Генерация суммарной частоты, как и генерация второй гармоники, активно используется при исследовании поверхностей и тонких слоев. Это объясняется тем, что, согласно дипольной модели, нелинейный сигнал генерируется преимущественно от объектов, не обладающих свойством центросимметричности [1], а значит, генерация в объеме отсутствует.

Как правило, исследование поверхностей происходит с помощью экспериментального определения пространственного распределения генерируемого излучения. Сопоставляя полученное пространственное распределение с предсказанным теоретической моделью, можно оценить значения коэффициентов анизотропии для исследуемой поверхности. Поскольку зачастую нелинейные свойства частицы обусловлены свойствами адсорбированного на ее поверхности красителя [2-7], то знание коэффициентов анизотропии позволяет определить поверхностную плотность адсорбированного вещества, свободную энергию [8], ориентацию молекул красителя на поверхности [9] и другие свойства.

\section{Функции, характеризующие пространственное распределение гармоники суммарной частоты}

Пусть на сферическую диэлектрическую частицу, покрытую тонким оптически нелинейным слоем и помещенную в диэлектрик, падают две плоские электромаг- нитные эллиптически поляризованные волны (рис. 1). Введем сферическую систему координат $(r, \theta, \varphi)$ с базисными векторами $\left(\mathbf{e}_{r}, \mathbf{e}_{\theta}, \mathbf{e}_{\varphi}\right)$ и началом координат в центре диэлектрической частицы. Зададим уравнения для векторов электрической напряженности падающих волн:

$$
\begin{aligned}
& \mathbf{E}^{(1)}(\mathbf{x})=E_{1} \mathbf{e}^{(1)} \exp \left(i \mathbf{k}^{(1)} \mathbf{x}-i \omega_{1} t\right), \\
& \mathbf{E}^{(2)}(\mathbf{x})=E_{2} \mathbf{e}^{(2)} \exp \left(i \mathbf{k}^{(2)} \mathbf{x}-i \omega_{2} t\right),
\end{aligned}
$$

где $E_{1}, E_{2}$ - комплексные амплитуды падающих волн, $\mathbf{e}^{(1)}, \mathbf{e}^{(2)}$ - единичные векторы, характеризующие поляризацию падающих волн, $\mathbf{k}^{(1)}, \mathbf{k}^{(2)}$ - волновые векторы падающих волн, $\omega_{1}, \omega_{2}$ - циклические частоты падающего излучения (в дальнейшем временная часть будет опущена).

В используемой нами дипольной модели в приближении Релея-Ганса-Дебая компоненты вектора нелинейной поляризации второго порядка задаются формулой

$$
P_{i}^{(2)}=\chi_{i j k}^{(2)} E_{j}^{(1)} E_{k}^{(2)}
$$

где $\chi_{i j k}^{(2)}$ - тензор нелинейной диэлектрической восприимчивости второго порядка для участка поверхности:

$$
\begin{aligned}
\chi_{i j k}^{(2)}= & \chi_{1}^{(2)} n_{i} n_{j} n_{k}+\chi_{2}^{(2)} n_{i} \delta_{j k}+\chi_{3}^{(2)} n_{j} \delta_{k i}+\chi_{4}^{(2)} n_{k} \delta_{i j} \\
& +\chi_{5}^{(2)} n_{m} n_{i} \varepsilon_{m j k}+\chi_{6}^{(2)} n_{m} n_{k} \varepsilon_{i j m}+\chi_{7}^{(2)} n_{m} n_{j} \varepsilon_{i m k},
\end{aligned}
$$

включающий коэффициенты анизотропии $\chi_{1-7}^{(2)}$, компоненты единичного вектора нормали к поверхности $n_{i}$, символ Кронекера $\delta_{i j}$ и символ Леви-Чивита $\varepsilon_{i j k}$. 


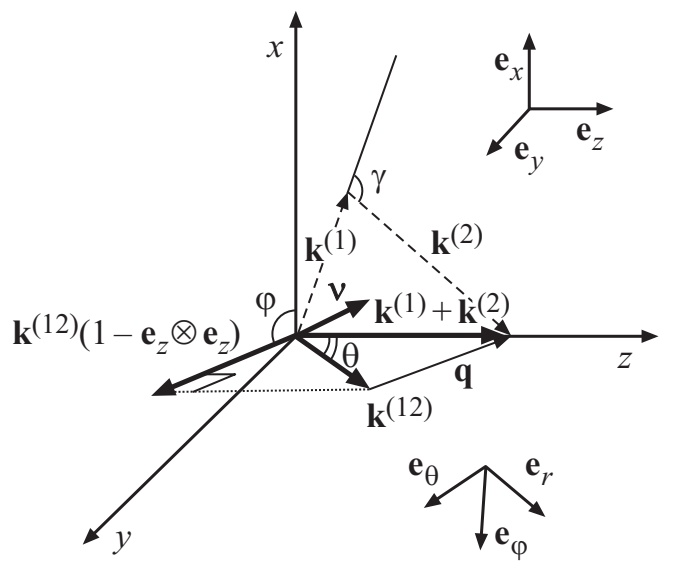

Рис. 1. Схема векторов, используемых в задаче.

Тогда вектор напряженности электрического поля гармоники суммарной частоты и радиальная составляющая вектора Умова-Пойнтинга в дальней зоне запишутся в виде (см. первую часть настоящей работы [10])

$$
\begin{gathered}
\mathbf{E}^{(12)}(\mathbf{x})=4 \pi \mu_{12} \frac{\left(\omega_{12}\right)^{2}}{c^{2}} \frac{\exp \left(i k_{12} r\right)}{r} \\
\times d_{0} a^{2} E_{1} E_{2}\left(1-\mathbf{e}_{r} \otimes \mathbf{e}_{r}\right) \mathbf{f}^{(12)}, \\
S_{r}^{(12)}(\mathbf{x})=\frac{c}{8 \pi} \frac{n_{12}}{\mu_{12}}\left|\mathbf{E}^{(12)}(\mathbf{x})\right|^{2},
\end{gathered}
$$

где

$$
\begin{aligned}
& \mathbf{f}^{(12)}=i \chi_{1}^{(2)}\left(-j_{3}(q a) \boldsymbol{v}\left(\boldsymbol{v} \mathbf{e}^{(1)}\right)\left(\boldsymbol{v} \mathbf{e}^{(2)}\right)\right. \\
& +\frac{1}{5}\left(j_{1}(q a)+j_{3}(q a)\right)\left(\boldsymbol{v}\left(\mathbf{e}^{(1)} \mathbf{e}^{(2)}\right)+\mathbf{e}^{(2)}\left(\boldsymbol{v} \mathbf{e}^{(1)}\right)\right. \\
& \left.\left.+\mathbf{e}^{(1)}\left(\boldsymbol{v} \mathbf{e}^{(2)}\right)\right)\right)+i j_{1}(q a)\left(\chi_{2}^{(2)} \boldsymbol{v}\left(\mathbf{e}^{(1)} \mathbf{e}^{(2)}\right)\right. \\
& \left.+\chi_{3}^{(2)} \mathbf{e}^{(2)}\left(\boldsymbol{v} \mathbf{e}^{(1)}\right)+\chi_{4}^{(2)} \mathbf{e}^{(1)}\left(\boldsymbol{v} \mathbf{e}^{(2)}\right)\right) \\
& +\frac{1}{3}\left(j_{0}(q a)+j_{2}(q a)\right)\left(\chi_{5}^{(2)}+\chi_{6}^{(2)}+\chi_{7}^{(2)}\right)\left[\mathbf{e}^{(1)} \times \mathbf{e}^{(2)}\right] \\
& -j_{2}(q a)\left(\chi_{5}^{(2)} \boldsymbol{v}\left(\boldsymbol{v}\left[\mathbf{e}^{(1)} \times \mathbf{e}^{2}\right]\right)\right. \\
& \left.+\chi_{6}^{(2)}\left[\mathbf{e}^{(1)} \times \boldsymbol{v}\right]\left(\boldsymbol{v} \mathbf{e}^{(2)}\right)+\chi_{7}^{(2)}\left[\boldsymbol{v} \times \mathbf{e}^{(2)}\right]\left(\boldsymbol{v} \mathbf{e}^{(1)}\right)\right) .
\end{aligned}
$$

Здесь $\mu_{12}$ и $n_{12}$ - магнитная проницаемость среды и показатель преломления на суммарной частоте $\omega_{12}=\omega_{1}+\omega_{2}, k_{12}-$ модуль волнового вектора генерируемой волны, $d_{0}$ и $a-$ толщина и радиус сферического нелинейного слоя соответственно, символ $\otimes$ означает тензорное произведение, $i$ вне индексов мнимая единица, конструкция $[\mathbf{a} \times \mathbf{b}]$ означает векторное произведение, $j_{m}(z)$ - сферические функции Бесселя порядка $m, v$ - единичный вектор вдоль вектора рассеяния q:

$$
\mathbf{q}=\mathbf{k}^{(1)}+\mathbf{k}^{(2)}-\mathbf{k}^{(12)}, \quad \boldsymbol{v}=\mathbf{q} /|\mathbf{q}| .
$$

При малых значениях радиуса сферического слоя $(q a \ll 1)$ получаем следующее выражение для вектоpa $\mathbf{f}^{(12)}$ :

$$
\begin{aligned}
& \mathbf{f}^{(12)}=i \frac{q a}{15} \chi_{1}^{(2)}\left(\boldsymbol{v}\left(\mathbf{e}^{(1)} \mathbf{e}^{(2)}\right)+\mathbf{e}^{(2)}\left(\boldsymbol{v} \mathbf{e}^{(1)}\right)+\mathbf{e}^{(1)}\left(\boldsymbol{v} \mathbf{e}^{2}\right)\right) \\
& +i \frac{q a}{3}\left(\chi_{2}^{(2)} \boldsymbol{v}\left(\mathbf{e}^{(1)} \mathbf{e}^{(2)}\right)+\chi_{3}^{(2)} \mathbf{e}^{(2)}\left(\boldsymbol{v} \mathbf{e}^{(1)}\right)+\chi_{4}^{(2)} \mathbf{e}^{(1)}\left(\boldsymbol{v} \mathbf{e}^{(2)}\right)\right) \\
& +\frac{1}{3}\left(\chi_{5}^{(2)}+\chi_{6}^{(2)}+\chi_{7}^{(2)}\right)\left[\mathbf{e}^{(1)} \times \mathbf{e}^{(2)}\right]
\end{aligned}
$$

при больших значениях радиуса сферического слоя $(q a \gg 1)$ получаем выражение

$$
\begin{aligned}
& \mathbf{f}^{(12)}=-\frac{\cos (q a)}{q a} i\left[\chi_{1}^{(2)} \boldsymbol{v}\left(\boldsymbol{v} \mathbf{e}^{(1)}\right)\left(\boldsymbol{v} \mathbf{e}^{(2)}\right)+\chi_{2}^{(2)} \boldsymbol{v}\left(\mathbf{e}^{(1)} \mathbf{e}^{(2)}\right)\right. \\
& \left.+\chi_{3}^{(2)} \mathbf{e}^{(2)}\left(\boldsymbol{v} \mathbf{e}^{(1)}\right)+\chi_{4}^{(2)} \mathbf{e}^{(1)}\left(\boldsymbol{v} \mathbf{e}^{(2)}\right)\right] \\
& +\frac{\sin (q a)}{q a}\left(\chi_{5}^{(2)} \boldsymbol{v}\left(\boldsymbol{v}\left[\mathbf{e}^{(1)} \times \mathbf{e}^{(2)}\right]\right)+\chi_{6}^{(2)}\left[\mathbf{e}^{(1)} \times \boldsymbol{v}\right]\left(\boldsymbol{v} \mathbf{e}^{(2)}\right)\right. \\
& \left.+\chi_{7}^{(2)}\left[\boldsymbol{v} \times \mathbf{e}^{(2)}\right]\left(\boldsymbol{v} \mathbf{e}^{(1)}\right)\right) .
\end{aligned}
$$

\section{Явный вид векторов в задаче}

Введем декартову систему координат. Пусть ось $O x$ направлена вверх, ось $O z$ направлена вправо вдоль вектора, равного сумме волновых векторов падающих волн $\mathbf{k}^{(1)}+\mathbf{k}^{(2)}$, а сами волновые векторы расположены в плоскости $O y z$ (рис. 1). Обозначим угол между $\mathbf{k}^{(1)}$ и $\mathbf{k}^{(2)}$ буквой $\gamma$ (угол раскрытия — „opening“ angle [11]). Тогда углы между волновыми векторами и осью $O z$ равны соответственно

$$
\gamma_{1}=\arccos \left(\frac{k_{1}+k_{2} \cos \gamma}{\sqrt{k_{1}^{2}+k_{2}^{2}+2 k_{1} k_{2} \cos \gamma}}\right)
$$

$$
\gamma_{2}=\arccos \left(\frac{k_{2}+k_{1} \cos \gamma}{\sqrt{k_{1}^{2}+k_{2}^{2}+2 k_{1} k_{2} \cos \gamma}}\right), \quad \gamma=\gamma_{1}+\gamma_{2} \text {, }
$$

где $k_{1}=\left|\mathbf{k}^{(1)}\right|$ и $k_{2}=\left|\mathbf{k}^{(2)}\right|-$ модули соответствующих волновых векторов. Если $k_{1}^{2}+k_{2}^{2}+2 k_{1} k_{2} \cos \gamma=0$, то $\gamma_{1}=0, \gamma_{2}=\gamma-\gamma_{1}$ 
Запишем выражения для волновых векторов и векторов поляризации падающих волн:

$$
\begin{gathered}
\mathbf{k}^{(1)}=k_{1}\left(-\sin \gamma_{1} \mathbf{e}_{y}+\cos \gamma_{1} \mathbf{e}_{z}\right), \\
\mathbf{k}^{(2)}=k_{2}\left(\sin \gamma_{2} \mathbf{e}_{y}+\cos \gamma_{2} \mathbf{e}_{z}\right), \\
+\frac{\left(\cos \varphi_{\text {in }}^{(1)}-i \sigma_{1} \sin \varphi_{\text {in }}^{(1)}\right) \mathbf{e}_{x}}{\sqrt{1+\sigma_{1}^{2}}} \\
+\frac{\cos \gamma_{1}\left(i \sigma_{1} \cos \varphi_{\text {in }}^{(1)}+\sin \varphi_{\text {in }}^{(1)}\right) \mathbf{e}_{y}}{\sqrt{1+\sigma_{1}^{2}}} \\
+\frac{\sin \gamma_{1}\left(i \sigma_{1} \cos \varphi_{\text {in }}^{(1)}+\sin \varphi_{\text {in }}^{(1)}\right) \mathbf{e}_{z}}{\sqrt{1+\sigma_{1}^{2}}}, \\
\mathbf{e}^{(2)}=\frac{\left(\cos \varphi_{\text {in }}^{(2)}-i \sigma_{2} \sin \varphi_{\text {in }}^{(2)}\right) \mathbf{e}_{x}}{\sqrt{1+\sigma_{2}^{2}}} \\
+\frac{\cos \gamma_{2}\left(i \sigma_{2} \cos \varphi_{\text {in }}^{(2)}+\sin \varphi_{\text {in }}^{(2)}\right) \mathbf{e}_{y}}{\sqrt{1+\sigma_{2}^{2}}} \\
-\frac{\sin \gamma_{2}\left(i \sigma_{2} \cos \varphi_{\text {in }}^{(2)}+\sin \varphi_{\text {in }}^{(2)}\right) \mathbf{e}_{z}}{\sqrt{1+\sigma_{2}^{2}}} .
\end{gathered}
$$

Здесь $\mathbf{e}_{x}, \mathbf{e}_{y}, \mathbf{e}_{z}-$ базисные векторы декартовой системы координат, $\sigma_{1}, \sigma_{2}-$ величины, характеризующие степень эллиптичности падающего излучения $(\sigma>0-$ правая поляризация, $\sigma<0-$ левая поляризация, $\sigma=0$ - линейная поляризация) и равные отношению меньшей полуоси эллипса поляризации к большей полуоси, $\varphi_{\text {in }}^{(1)}, \varphi_{\text {in }}^{(2)}$ - углы между осью $O x$ и большими полуосями эллипсов поляризации соответствующих падающих электромагнитных волн.

Тогда вектор рассеяния будет иметь вид $\mathbf{q}=\mathbf{k}^{(1)}+\mathbf{k}^{(2)}-\mathbf{k}^{(12)}=-\left[\left(k_{12}-\zeta \cos \theta\right) \mathbf{e}_{r}+\zeta \sin \theta \mathbf{e}_{\theta}\right]$,

$$
|\mathbf{q}|=\sqrt{\xi^{2}-2 \xi k_{12} \cos \theta+k_{12}^{2}},
$$

где

$$
\xi=\left|\mathbf{k}^{(1)}+\mathbf{k}^{(2)}\right|=\sqrt{k_{1}^{2}+k_{2}^{2}+2 k_{1} k_{2} \cos \gamma}
$$

- вспомогательная величина, равная модулю суммы волновых векторов падающих волн. Учет дисперсии, характеризуемой коэффициентом

$$
\xi=\frac{k_{12}}{k_{1}+k_{2}}=\frac{n_{12} \omega_{12}}{n_{1} \omega_{1}+n_{2} \omega_{2}},
$$

в такой модели происходит путем задания модуля волнового вектора генерируемой волны $k_{12}$. Тогда единичный вектор вдоль вектора рассеяния запишется как

$$
\begin{gathered}
\boldsymbol{v}=\frac{\mathbf{q}}{|\mathbf{q}|}=\frac{\left(-k_{12}+\xi \cos \theta\right) \mathbf{e}_{r}-\xi \sin \theta \mathbf{e}_{\theta}}{\sqrt{\xi^{2}-2 \xi k_{12} \cos \theta+k_{12}^{2}}}, \\
\boldsymbol{v}=0, \quad \text { если } \xi^{2}-2 \xi k_{12} \cos \theta+k_{12}^{2}=0 .
\end{gathered}
$$

\section{Свойства функций, характеризующих пространственное распределение поля суммарной частоты}

Подставляя выражения (9)-(14) в формулы (4)-(6), можем обнаружить следующие математические свойства вектора $\mathbf{f}^{(12)}(\theta, \varphi)$ и функции $S_{r}^{(12)}(\theta, \varphi)$ : для любых значений параметров задачи $\left(m_{1}, m_{2}\right.$ - целые)

$$
\begin{gathered}
\mathbf{f}^{(12)}\left(\theta+2 \pi m_{1}, \varphi+2 \pi m_{2}\right)=\mathbf{f}^{(12)}(\theta, \varphi), \\
\mathbf{f}^{(12)}(-\theta, \varphi)=-\left(1-2 \mathbf{e}_{r} \otimes \mathbf{e}_{r}\right) \mathbf{f}^{(12)}(\theta, \varphi+\pi), \\
S_{r}^{(12)}\left(\theta+2 \pi m_{1}, \varphi+2 \pi m_{2}\right)=S_{r}^{(12)}(\theta, \varphi), \\
S_{r}^{(12)}(-\theta, \varphi)=S_{r}^{(12)}(\theta, \varphi+\pi) .
\end{gathered}
$$

Свойства (15) выполняются также для генерации второй гармоники от нелинейного слоя на поверхности сферической частицы [12] и для генерации суммарной частоты от нелинейного слоя на поверхности цилиндрической частицы [13]. Если $\left|\sigma_{1}\right|=1$ и $\left|\sigma_{2}\right|=1$, то

$$
\begin{gathered}
i\left(1-(1-i) \mathbf{e}_{\theta} \otimes \mathbf{e}_{\theta}\right) \mathbf{f}^{(12)}(-\theta, \varphi)=\left[i\left(1-(1-i) \mathbf{e}_{\theta} \otimes \mathbf{e}_{\theta}\right)\right. \\
\left.\times \mathbf{f}^{(12)}(\theta,-\varphi) \exp \left(2 i\left(\sigma_{1} \varphi_{\text {in }}^{(1)}+\sigma_{2} \varphi_{\text {in }}^{(2)}\right)\right)\right]^{*} \\
S_{r}^{(12)}(-\theta, \varphi)=S_{r}^{(12)}(\theta,-\varphi) .
\end{gathered}
$$

В случае, когда $\varphi_{\text {in }}^{(1)}=\pi m_{1}$ и $\varphi_{\text {in }}^{(2)}=\pi m_{2}$ или $\varphi_{\text {in }}^{(1)}=$ $=\pi m_{1}+\pi / 2$ и $\varphi_{\text {in }}^{(2)}=\pi m_{2}+\pi / 2, m_{1}, m_{2}$ - целые, имеем

$$
\begin{aligned}
i(1- & \left.(1-i) \mathbf{e}_{\theta} \otimes \mathbf{e}_{\theta}\right) \mathbf{f}^{(12)}(-\theta, \varphi) \\
= & {\left[i\left(1-(1-i) \mathbf{e}_{\theta} \otimes \mathbf{e}_{\theta}\right) \mathbf{f}^{(12)}(\theta,-\varphi)\right]^{*}, } \\
& S_{r}^{(12)}(-\theta, \varphi)=S_{r}^{(12)}(\theta,-\varphi) .
\end{aligned}
$$

Если $\chi_{2}^{(2)} \neq 0, \chi_{1,3-7}^{(2)}=0$, получаем

$$
\begin{gathered}
\mathbf{e}_{\varphi} \mathbf{f}^{(12)}(\theta, \varphi)=0, \quad \mathbf{f}^{(12)}(\theta, \varphi+\Delta \varphi)=\mathbf{f}^{(12)}(\theta, \varphi), \\
S_{r}^{(12)}(\theta, \varphi+\Delta \varphi)=S_{r}^{(12)}(\theta, \varphi), \quad \forall \Delta \varphi .
\end{gathered}
$$

Когда угол раскрытия $\gamma=0$ или $\gamma=\pi$, выполняются следующие свойства:

$$
\begin{gathered}
\mathbf{f}^{(12)}(\theta, \varphi+\pi m)=\mathbf{f}^{(12)}(\theta, \varphi), \\
S_{r}^{(12)}(\theta, \varphi)=S_{r}^{(12)}(\theta, \varphi+\pi m), \quad m \text { - целое. }
\end{gathered}
$$

В случае $\gamma=0$ или $\gamma=\pi, \varphi_{\text {in }}^{(2)}-(-1)^{\gamma / \pi} \varphi_{\text {in }}^{(1)}=m \pi / 2$, $m$ - целое, имеем

$$
\begin{aligned}
& \operatorname{Re}\left[\left(1-(1-i) \mathbf{e}_{\varphi} \otimes \mathbf{e}_{\varphi}\right) \mathbf{f}^{(12)}(\theta, \varphi+\pi / 2) \exp \left(i m \frac{\pi}{2}\right)\right] \\
& \quad=-\operatorname{Re}\left[\left(1-(1-i) \mathbf{e}_{\varphi} \otimes \mathbf{e}_{\varphi}\right) \mathbf{f}^{(12)}(\theta, \varphi) \exp \left(i m \frac{\pi}{2}\right)\right]
\end{aligned}
$$


Для некирального слоя при $\gamma=0, \pi, \sigma_{1}= \pm \sigma_{2}, \chi_{1-4}^{(2)} \neq 0$, $\chi_{5-7}^{(2)}=0, m$ - целое, выполняется равенство

$\operatorname{Re}\left[\mathbf{f}^{(12)}(\theta, \varphi+\pi / 2)\right]=-\frac{\sigma_{1}}{\sigma_{2}}(-1)^{\gamma / \pi} \operatorname{Re}\left[\mathbf{f}^{(12)}(\theta, \varphi+m \pi)\right]$.

Для кирального слоя при $\gamma=0, \pi, \sigma_{1}= \pm \sigma_{2}, \chi_{1-4}^{(2)}=0$, $\chi_{5-7}^{(2)} \neq 0, m-$ целое, имеем

$\operatorname{Im}\left[\mathbf{f}^{(12)}(\theta, \varphi+\pi / 2)\right]=-\frac{\sigma_{1}}{\sigma_{2}}(-1)^{\gamma / \pi} \operatorname{Im}\left[\mathbf{f}^{(12)}(\theta, \varphi+m \pi)\right]$.

При аксиальной симметрии задачи, когда $\gamma=0, \pi$, $\left|\sigma_{1}\right|=\left|\sigma_{2}\right|=1$, получаем свойства

$$
\begin{gathered}
\mathbf{f}^{(12)}(\theta, \varphi+\Delta \varphi)=\mathbf{f}^{(12)}(\theta, \varphi) \\
\times \exp \left(i \frac{k_{1}+(-1)^{\gamma / \pi} k_{2}}{\left|k_{1}+(-1)^{\gamma / \pi} k_{2}\right|}\left(\sigma_{1}+(-1)^{\gamma / \pi} \sigma_{2}\right) \Delta \varphi\right), \\
S_{r}^{(12)}(\theta, \varphi+\Delta \varphi)=S_{r}^{(12)}(\theta, \varphi), \quad \forall \Delta \varphi .
\end{gathered}
$$

При зеркальной симметрии задачи, когда $\gamma=0, \pi$, $\varphi_{\text {in }}^{(2)}-(-1)^{\gamma / \pi} \varphi_{\text {in }}^{(1)}=m_{1} \pi / 2, m_{1}, m_{2}$ - целые, $m=1,2$,

$$
\begin{aligned}
i(1 & \left.-(1-i) \mathbf{e}_{\varphi} \otimes \mathbf{e}_{\varphi}\right) \mathbf{f}^{(12)}\left(\theta, \pi m_{2}-\varphi+(-1)^{m \gamma / \pi}\right. \\
& \left.\times \frac{(-1)^{\gamma / \pi} k_{1}+k_{2}}{\left|(-1)^{\gamma / \pi} k_{1}+k_{2}\right|} \varphi_{\text {in }}^{(m)}\right) \exp \left(i m_{1} \pi / 2\right) \\
& =\left[i ( 1 - ( 1 - i ) \mathbf { e } _ { \varphi } \otimes \mathbf { e } _ { \varphi } ) \mathbf { f } ^ { ( 1 2 ) } \left(\theta, \varphi+(-1)^{m \gamma / \pi}\right.\right. \\
& \left.\left.\times \frac{(-1)^{\gamma / \pi} k_{1}+k_{2}}{\left|(-1)^{\gamma / \pi} k_{1}+k_{2}\right|} \varphi_{\text {in }}^{(m)}\right) \exp \left(i m_{1} \pi / 2\right)\right]^{*}, \\
S_{r}^{(12)}( & \left.\theta, \pi m_{2}-\varphi+(-1)^{m \gamma / \pi} \frac{(-1)^{\gamma / \pi} k_{1}+k_{2}}{\left|(-1)^{\gamma / \pi} k_{1}+k_{2}\right|} \varphi_{\text {in }}^{(m)}\right) \\
= & S_{r}^{(12)}\left(\theta, \varphi+(-1)^{m \gamma / \pi} \frac{(-1)^{\gamma / \pi} k_{1}+k_{2}}{\left|(-1)^{\gamma / \pi} k_{1}+k_{2}\right|} \varphi_{\text {in }}^{(m)}\right) .
\end{aligned}
$$

Для произвольных углов $\varphi_{\text {in }}^{(1)}, \varphi_{\text {in }}^{(2)}$ при $\gamma=0, \pi, m-$ целое, получаем

$$
\begin{aligned}
& \operatorname{Im}\left[( 1 - ( 1 - i ) \mathbf { e } _ { \varphi } \otimes \mathbf { e } _ { \varphi } ) \mathbf { f } ^ { ( 1 2 ) } \left(\theta, \frac{\pi m}{2}-\varphi\right.\right. \\
& \left.\left.+\frac{k_{1}+(-1)^{\gamma / \pi} k_{2}}{\left|k_{1}+(-1)^{\gamma / \pi} k_{2}\right|}\left(\varphi_{\text {in }}^{(1)}+(-1)^{\gamma / \pi} \varphi_{\text {in }}^{(2)}\right) / 2\right) \exp \left(i \frac{\pi m}{2}\right)\right] \\
& =\operatorname{Im}\left[( 1 - ( 1 - i ) \mathbf { e } _ { \varphi } \otimes \mathbf { e } _ { \varphi } ) \mathbf { f } ^ { ( 1 2 ) } \left(\theta, \varphi+\frac{k_{1}+(-1)^{\gamma / \pi} k_{2}}{\left|k_{1}+(-1)^{\gamma / \pi} k_{2}\right|}\right.\right. \\
& \left.\left.\times\left(\varphi_{\text {in }}^{(1)}+(-1)^{\gamma / \pi} \varphi_{\text {in }}^{(2)}\right) / 2\right) \exp \left(i \frac{\pi m}{2}\right)\right]
\end{aligned}
$$

Если выполняются условия $\gamma=0, \pi, \varphi_{\text {in }}^{(1)}+(-1)^{\gamma / \pi} \varphi_{\text {in }}^{(2)}=$ $=m_{1} \pi / 2, m_{1}, m_{2}$ - целые, то верны также свойства

$$
\begin{aligned}
\operatorname{Im} & {\left[\left(1-(1-i) \mathbf{e}_{\varphi} \otimes \mathbf{e}_{\varphi}\right) \mathbf{f}^{(12)}\left(\theta, m_{2} \pi / 2-\varphi\right)\right.} \\
& \left.\times \exp \left(i\left(m_{1}+m_{2}\right) \pi / 2\right)\right]=\operatorname{Im}\left[\left(1-(1-i) \mathbf{e}_{\varphi} \otimes \mathbf{e}_{\varphi}\right)\right. \\
& \left.\times \mathbf{f}^{(12)}(\theta, \varphi) \exp \left(i\left(m_{1}+m_{2}\right) \pi / 2\right)\right]
\end{aligned}
$$

Для случая $\gamma=0, \pi, \varphi_{\text {in }}^{(1)}=m_{1} \pi / 2, \varphi_{\text {in }}^{(2)}=m_{2} \pi / 2\left(m_{1}\right.$, $m_{2}, m_{3}, m_{4}$ - целые) выполняются дополнительные свойства:

$$
\begin{aligned}
& \left(1-(1-i) \mathbf{e}_{\varphi} \otimes \mathbf{e}_{\varphi}\right) \mathbf{f}^{(12)}\left(\theta, m_{3} \pi / 2+m_{4} \pi-\varphi\right) \\
& \quad \times \exp \left(i\left(m_{1}+m_{2}+1\right) \pi / 2\right)=\left[\left(1-(1-i) \mathbf{e}_{\varphi} \otimes \mathbf{e}_{\varphi}\right)\right. \\
& \left.\quad \times \mathbf{f}^{(12)}\left(\theta, m_{3} \pi / 2+\varphi\right) \exp \left(i\left(m_{1}+m_{2}+1\right) \pi / 2\right)\right]^{*}, \\
& S_{r}^{(12)}\left(\theta, m_{3} \pi / 2+m_{4} \pi-\varphi\right)=S_{r}^{(12)}\left(\theta, m_{3} \pi / 2+\varphi\right) .
\end{aligned}
$$

\section{Графический анализ}

Построим диаграммы направленности для следующих значений параметров (рис. 2,3):

$$
\begin{gathered}
k_{1} a=0.1, \quad k_{2} a=0.1, \quad \gamma=0.5, \\
\varphi_{\text {in }}^{(1)}=1, \quad \varphi_{\text {in }}^{(2)}=-1, \quad \sigma_{1}=0.5, \quad \sigma_{2}=0.5 .
\end{gathered}
$$

На диаграммах направленности для малого размера частицы можно видеть, что их форма сильно зависит от типа анизотропии, присущего поверхности.

Для типа анизотропии $\chi_{1}^{(2)} \neq 0$ (рис. 2,a) видим наличие двух направлений преимущественного излучения, причем увеличение угла раскрытия (рис. 3,a) ведет к смещению главных лепестков диаграммы направленности в сторону, противоположную $\mathbf{k}^{(1)}+\mathbf{k}^{(2)}$. В сторону вектора $\mathbf{k}^{(1)}+\mathbf{k}^{(2)}$ излучается волна правой поляризации, а в противоположном направлении - левой.

В случае $\chi_{2}^{(2)} \neq 0$ (рис. $2, b$ и $3, b$ ) диаграммы направленности имеют ось симметрии (согласно свойству (18)), направленную вдоль вектора $\mathbf{k}^{(1)}+\mathbf{k}^{(2)}$, поляризация генерируемого излучения линейная. Увеличение угла раскрытия никак не влияет на вид диаграммы направленности. Рассматривая формулу для малого радиуса частицы (7), видим, что диаграмму направленности формирует слагаемое $i \frac{q a}{3} \chi_{2}^{(2)} \boldsymbol{v}\left(\mathbf{e}^{(1)} \mathbf{e}^{(2)}\right)$. Здесь скалярное произведение векторов поляризации одинаково во всех направлениях. Вектор v имеет вид (14), и его радиальная составляющая пропадает после подстановки $\mathbf{f}^{(12)}$ в формулу (4). Остается слагаемое $\frac{-\xi \sin \theta \mathbf{e}_{\theta}}{\sqrt{\xi^{2}-2 \xi k_{12} \cos \theta+k_{12}^{2}}}$, не зависящее от угла $\varphi$, что приводит к осевой симметрии диаграммы направленности. Вектор напряженности электрического поля при этом направлен вдоль вектора $\mathbf{e}_{\theta}$, поэтому волна линейно поляризована. 


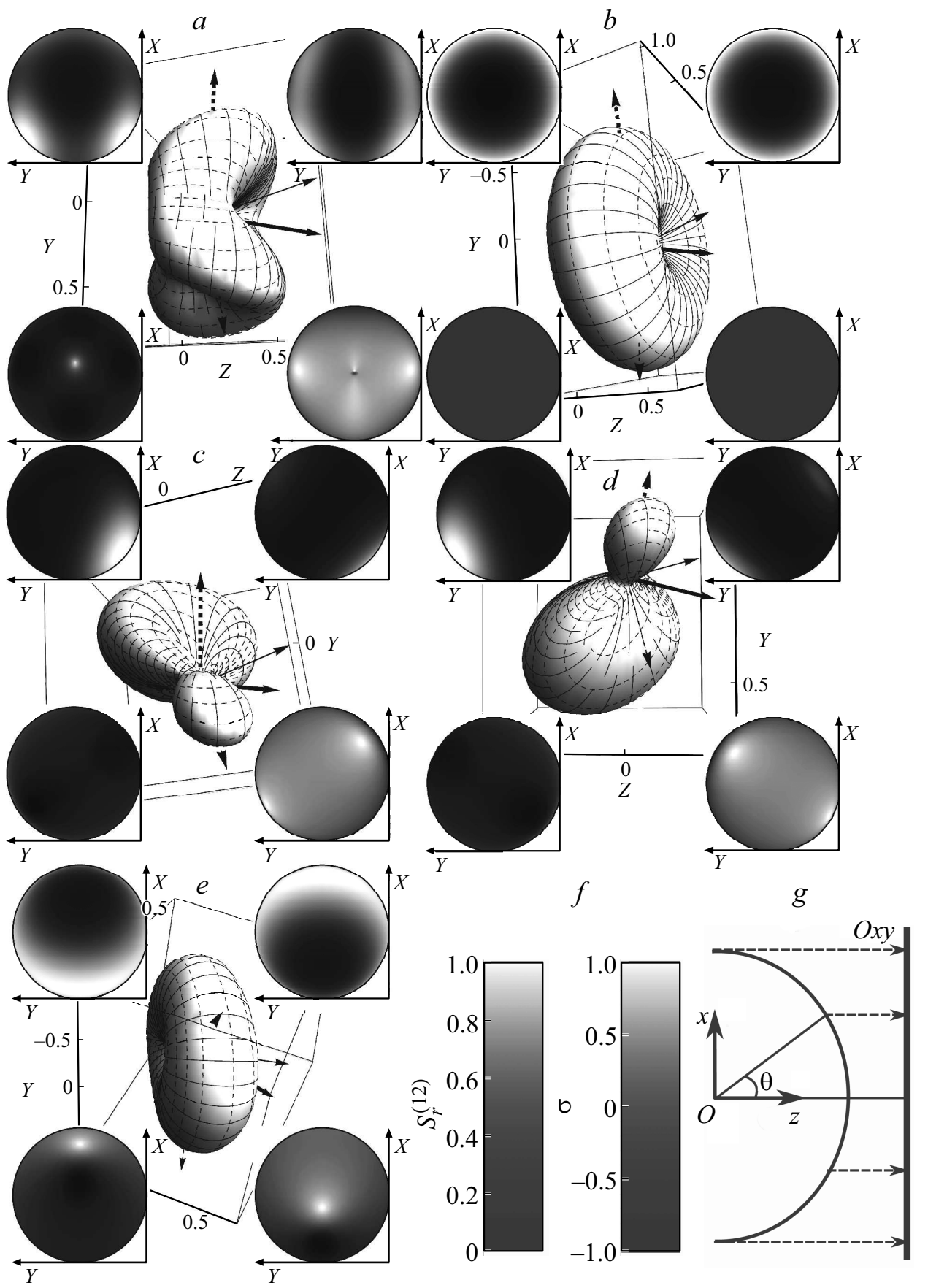

Рис. 2. Трехмерные нормированные диаграммы направленности для генерации суммарной частоты от сферического нелинейного слоя. Угол раскрытия $\gamma=0.5 \mathrm{rad}$. Сплошные стрелки показывают направления волновых векторов падающих волн. Штриховые стрелки направлены вдоль больших полуосей эллипсов поляризации падающих волн. Тонкие стрелки относятся к первой волне, утолщенные - ко второй. Сплошные линии, изображенные на поверхности диаграммы, направлены вдоль проекций больших полуосей эллипса поляризации излучения суммарной частоты на поверхность диаграммы направленности, а штриховые линии проекции меньшей полуоси эллипса поляризации излучения суммарной частоты на поверхность диаграммы направленности. В верхней части диаграммы направленности расположены ее проекции на плоскость $O x y$ (левая: $\pi / 2<\theta<\pi$, правая: $0<\theta<\pi / 2$ ), как показано на рис. $2, g$. Белый цвет соответствует максимальной мощности излучения в данном направлении, черный цвет соответствует отсутствию излучения в данном направлении. В нижней части диаграммы направленности расположены графики, характеризующие степень эллиптичности генерируемого излучения с тем же правилом проецирования. Белый цвет соотвествует право циркулярно поляризованному излучению, черный цвет соответствует лево циркулярно поляризованному излучению, серый цвет соответствует линейной поляризации излучения. Легенда представлена на рис. 2,f. Предполагается, что для каждого из рисунков все коэффициенты анизотропии, кроме указанных, равны нулю: $(a) \chi_{1}^{(2)} \neq 0,(b) \chi_{2}^{(2)} \neq 0,(c) \chi_{3}^{(2)} \neq 0$, (d) $\chi_{4} \neq 0,(e) \chi_{5}^{(2)} \neq 0, \chi_{6}^{(2)} \neq 0, \chi_{7}^{(2)} \neq 0$. 


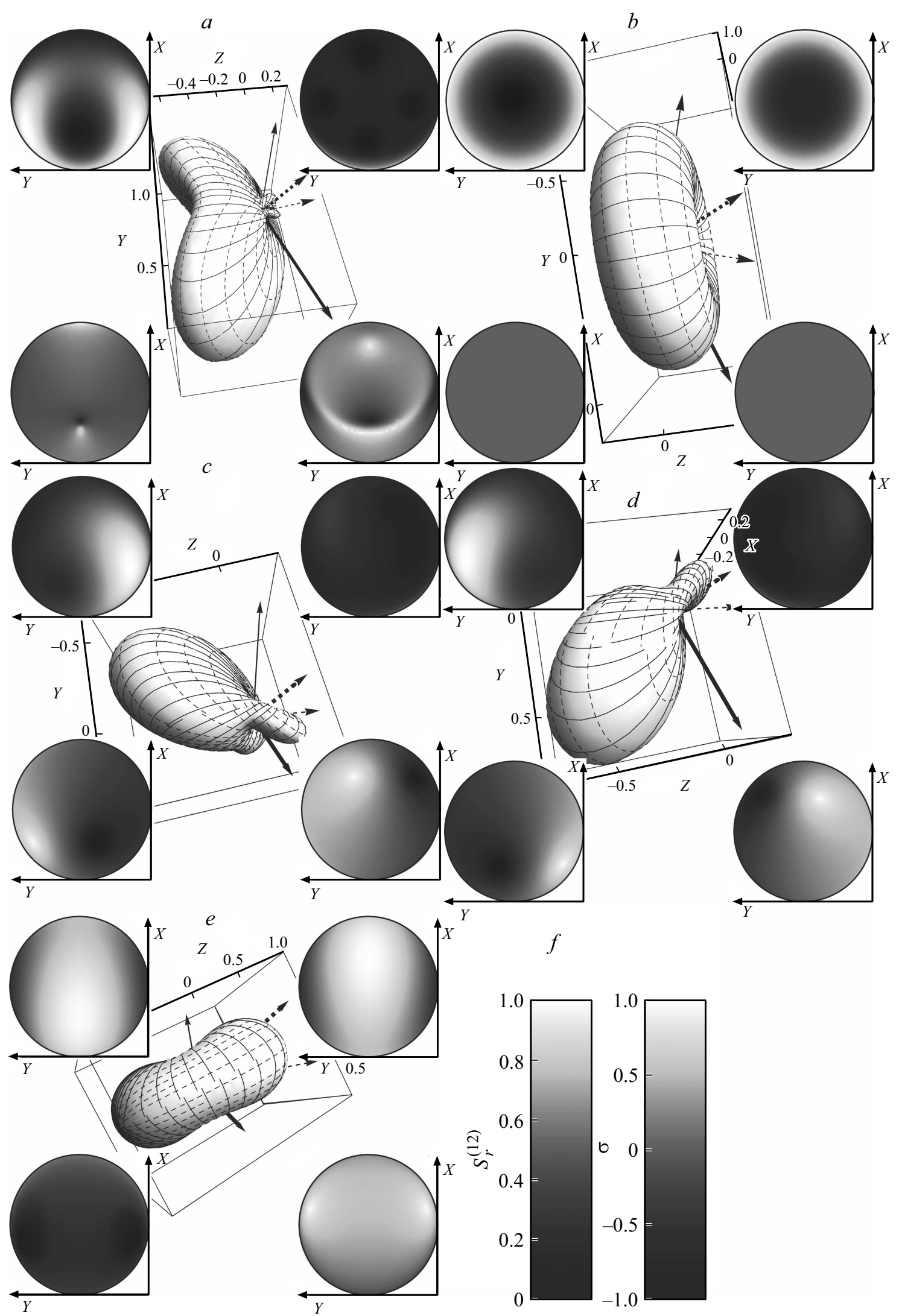

Pис. 3. Трехмерные нормированные диаграммы направленности для генерации суммарной частоты от сферического нелинейного слоя. Угол раскрытия $\gamma=2.5 \mathrm{rad}$. Обозначения такие же, как и на рис. 2. Коэффициенты анизотропии: $(a) \chi_{1}^{(2)} \neq 0,(b) \chi_{2}^{(2)} \neq 0$, (c) $\chi_{3}^{(2)} \neq 0,(d) \chi_{4} \neq 0,(e) \chi_{5}^{(2)} \neq 0, \chi_{6}^{(2)} \neq 0, \chi_{7}^{(2)} \neq 0$. Легенда на рис. $3, f$. 
В случаях $\chi_{3}^{(2)} \neq 0$ (рис. 2,c) и $\chi_{4}^{(2)} \neq 0$ (рис. $2, d$ ) имеем один ярко выраженный лепесток и противоположно направленный побочный. Побочный лепесток расположен ближе к вектору $\mathbf{k}^{(1)}+\mathbf{k}^{(2)}$, а главный - к противоположному вектору. Увеличение угла раскрытия (рис. 3, $c$ и 3,d) ведет к уменьшению побочного лепестка и увеличению главного. В случаях $\chi_{5}^{(2)} \neq 0, \chi_{6}^{(2)} \neq 0$, $\chi_{7}^{(2)} \neq 0$ (рис. 2, e и 3,e) получаем диаграммы направленности, напоминающие по форме повернутую диаграмму направленности для типа анизотропии $\chi_{2}^{(2)} \neq 0$. Различие между этими тремя случаями возникает только с увеличением радиуса нелинейного сферического слоя. Это объясняется тем, что для малых радиусов частицы диаграмма направленности определяется одним слагаемым из формулы (7): $\frac{1}{3}\left(\chi_{5}^{(2)}+\chi_{6}^{(2)}+\chi_{7}^{(2)}\right)\left[\mathbf{e}^{(1)} \times \mathbf{e}^{(2)}\right]$. В нем все коэффициенты $\chi_{5-7}^{(2)}$ оказывают одинаковое влияние на результат.

Стоит заметить, что если падающие волны имеют линейную поляризацию и нелинейный слой является киральным $\left(\chi_{1-4}^{(2)}=0, \forall \chi_{5-7}^{(2)}\right)$ или некиральным $\left(\forall \chi_{1-4}^{(2)}, \chi_{5-7}^{(2)}=0\right)$, то генерируется исключительно линейно поляризованное излучение.

\section{Сравнение с работами других авторов}

В работе [14] представлен частный случай нашей задачи: генерация гармоники суммарной частоты при отсутствии киральных элементов у тензора нелинейной диэлектрической восприимчивости. УГловые зависимости поля суммарной частоты приведены только для генерации в плоскости, содержащей векторы $\mathbf{k}^{(1)}$ и $\mathbf{k}^{(2)}$, причем рассмотрен только случай $\left|\mathbf{k}^{(1)}+\mathbf{k}^{(2)}\right|=\left|\mathbf{k}^{(12)}\right|$. Формулы в этом случае совпадают с частным случаем нашего решения, если исправить в их работе $\sigma^{3}$ на $\sigma^{2}$ (куб радиуса частицы на квадрат радиуса частицы) в выражении для $B$ (эта опечатка приводила к суммированию безразмерной и размерной величин). С учетом этого исправления связь обозначений в [14] с нашими следущая:

$$
\begin{gathered}
A(q, a)=4 i a^{2} \frac{1}{5}\left(j_{1}(q a)+j_{3}(q a)\right), \\
B(q, a)=4 i a^{2}\left(\frac{3}{10} j_{1}(q a)-\frac{1}{5} j_{3}(q a)\right) .
\end{gathered}
$$

В работе [1] используется тот же подход, что и в [14], но с учетом киральных элементов. Однако авторы [1] не указали временную зависимость $(\exp (-i \omega t)$ или $\exp (i \omega t))$. Если предполагается зависимость $\exp (-i \omega t)$, то в работе [1] потерян множитель $(-1)$ в векторе рассеяния q. Если предполагается зависимость $\exp (i \omega t)$, то в формуле для напряженности электрического поля суммарной частоты (5) из работы [1] в степени фазового множителя $e^{i k_{0} r_{0}}$ также отсутствует $(-1)$. Оба этих их варианта приводят к отличным от полученных в настоящей работе пространственным распределениям поля, генерируемого от нелинейного слоя, тензор нелинейной восприимчивости которого имеет киральные и некиральные коэффициенты, не равные нулю. Также в работе [1] присутствует неточность в записи коэффициента $\Gamma_{y z x}^{(2)}$ в формуле (13), где вместо слагаемого $2 \Lambda(0,0) \bar{\chi}_{r^{\prime} \theta^{\prime} \varphi^{\prime}}^{(2)}$ должно быть слагаемое $2 \Lambda(0,0) \bar{\chi}_{\varphi^{\prime} r^{\prime} \theta^{\prime}}^{(2)}$. Такая замена приведет к выполнению свойства симметрии относительно перестановки индексов, т.е. выражения для коэффициентов $\Gamma_{x y z}^{(2)}, \Gamma_{y z x}^{(2)}, \Gamma_{z x y}^{(2)}$ можно будет получить друг из друга циклической перестановкой соответствующих индексов. Только в таком случае результат [1] будет совпадать с полученным в нашей работе. Связь обозначений в статье [1] и нашей статье следущая:

$$
\begin{gathered}
\Lambda(0,0)(q, a)=2 a^{2} j_{0}(q a), \\
\Lambda(1,0)(q, a)=2 i a^{2} j_{1}(q a), \\
\Lambda(0,2)(q, a)=\frac{4}{3} a^{2}\left(j_{0}(q a)+j_{2}(q a)\right), \\
\Lambda(1,2)(q, a)=\frac{4}{5} i a^{2}\left(j_{1}(q a)+j_{3}(q a)\right) .
\end{gathered}
$$

В работе [11] описана генерация гармоники суммарной частоты от поверхности тел произвольной формы. В частности, получено аналитическое решение в виде рядов для поверхности, имеющей форму эллипсоида вращения. Частный случай этого решения (сферический слой) не совпадает с нашим, отличаясь множителем $(-1)$ в выражении для вектора рассеяния q. Угловое распределение гармоники суммарной частоты в [11] получено только в плоскости, содержащей волновые векторы падающих волн и только для случая $\left|\mathbf{k}^{(1)}+\mathbf{k}^{(2)}\right|=\left|\mathbf{k}^{(12)}\right|$. Предельные значения компонент тензора эффективной восприимчивости при малых радиусах сферического слоя совпадают с полученными в настоящей работе, за исключением $\mathbf{A}_{x^{\prime} x^{\prime} z^{\prime}}=i V q_{x^{\prime}} / 5$, который в нашем случае с их обозначениями принимает вид $i V q_{z^{\prime}} / 5$. Связь наших обозначений с обозначениями авторов работы [11] следущая:

$$
\begin{gathered}
\Gamma_{i j k}^{(2)}=a^{2} \mathbf{X}_{i j k}^{(12)} \\
A_{i j k}(\mathbf{q})=4 \pi a^{2} i\left[\frac{1}{5}\left(j_{1}(q a)+j_{3}(q a)\right)\right. \\
\left.\times\left(v_{i} \delta_{j k}+v_{j} \delta_{k i}+v_{k} \delta_{i j}\right)-j_{3}(q a) v_{i} v_{j} v_{k}\right], \\
B_{i j}(\mathbf{q})=4 \pi a^{2}\left[\delta_{i j} \frac{1}{3}\left(j_{0}(q a)+j_{2}(q a)\right)-v_{i} v_{j} j_{2}(q a)\right], \\
C_{k}(\mathbf{q})=4 \pi a^{2} i v_{k} j_{1}(q a) .
\end{gathered}
$$

Причем в таком виде свойства этих тензоров, приведенные для частиц произвольной формы в [11], также 
выполняются:

$$
\begin{gathered}
A_{j k k}=C_{j}, \\
C_{k}=i q_{k} \int_{V} \exp \left(i \mathbf{q} \mathbf{x}^{\prime}\right) d^{3} \mathbf{x}^{\prime} \\
=i q_{k} 4 \pi \int_{0}^{a} j_{0}(q r) r^{2} d r=i q_{k} 4 \pi a^{3} \frac{j_{1}(q a)}{q a}, \\
B_{k k}=\int_{S} \exp \left(i \mathbf{q} \mathbf{x}^{\prime}\right) d^{2} \mathbf{x}^{\prime} \\
=2 \pi a^{2} \int_{0}^{\pi} \exp (i q a \cos \theta) \sin \theta d \theta=4 \pi a^{2} j_{0}(q a),
\end{gathered}
$$

где повторяющиеся индексы означают свертку, $\int_{V} \exp \left(i \mathbf{q} \mathbf{x}^{\prime}\right) d^{3} \mathbf{x}^{\prime}$ - линейный объемный форм-фактор, a $\int_{S} \exp \left(i \mathbf{q} \mathbf{x}^{\prime}\right) d^{2} \mathbf{x}^{\prime}-$ линейный поверхностный формфактор [11].

\section{Заключение}

В настоящей работе на основе аналитического решения задачи о генерации суммарной частоты в приближении Релея-Ганса-Дебая, учитывающем дисперсию, показано, что при малых радиусах сферического слоя вид диаграммы направленности сильно зависит от типа анизотропии, присущего нелинейному слою, и от угла раскрытия между волновыми векторами падающих волн. При этом угол раскрытия не влияет на форму диаграммы направленности при одном из типов анизотропии. Для остальных типов он приводит к росту генерации в направлении, обратном сумме волновых векторов падающих волн.

Показано, что падение двух линейно поляризованных волн на нелинейный сферический слой, обладающий только киральными $\left(\chi_{1-4}^{(2)}=0, \forall \chi_{5-7}^{(2)}\right)$ или только некиральными $\left(\forall \chi_{1-4}^{(2)}, \chi_{5-7}^{(2)}=0\right)$ свойствами, приводит к генерации также линейно поляризованного излучения. Последнее свойство верно и для генерации второй гармоники от нелинейного слоя на поверхности сферической частицы, поскольку генерацию второй гармоники можно считать частным случаем рассмотренной задачи. Анализируемое решение описывает генерацию второй гармоники в случае нулевого угла раскрытия, при одинаковой поляризации, частоте и амплитуде падающих волн. Тогда остается лишь два варьируемых параметра: частота падающего излучения и его поляризация [12]. Явление генерации суммарной частоты имеет гораздо больше параметров, а потому позволяет извлечь больше информации из эксперимента.

\section{Список литературы}

[1] de Beer A.G.F., Roke S. // Phys. Rev. B. 2007. V. 75. N 24. P. 245438. doi 10.1103/PhysRevB.75.245438

[2] Wang H., Yan E.C.Y., Borguet E., Eisenthal K.B. // Chem. Phys. Lett. 1996. V. 259. N 1-2. P. 15. doi 10.1016/0009-2614(96)00707-5

[3] Yang N., Angerer W.E., Yodh A.G. // Phys. Rev. Lett. 2001. V. 87. N 10. P. 103902. doi 10.1103/PhysRevLett.87.103902

[4] Jen S.H., Dai H.L. // J. Phys. Chem. B. 2006. V. 110. N 46. P. 23000. doi 10.1021/jp0644762

[5] Jen S.-H., Gonella G., Dai H.-L. // J. Phys. Chem. A. 2009. V. 113. N 6. P. 4758. doi 10.1021/jp9009959

[6] Viarbitskaya S., Kapshai V., van der Meulen P., Hansson T. // Phys. Rev. A. 2010. V. 81. N 5. P. 053850. doi 10.1103/PhysRevA.81.053850

[7] Shan J., Dadap J.I., Stiopkin I., Reider G.A., Heinz T.F. // Phys. Rev. A. 2006. V. 73. N 2. P. 023819. doi 10.1103/PhysRevA.73.023819.

[8] Subir M., Liu J., Eisenthal K.B. // J. Phys. Chem. C. 2008. V. 112. N 40. P. 15809 . doi 10.1021/jp8047168

[9] de Beer A.G.F., Roke S. // J. Chem. Phys. 2010. V. 132. N 23. P. 234702. doi 10.1063/1.3429969

[10] Капшай В.Н., Шамына А.А. // Опт. и спектр. 2018. Т. 124. B. 6. C. 795-803; Kapshai V.N., Shamyna A.A. // Opt. Spectrosc. 2018. V. 124. N 6.

[11] de Beer A.G.F., Roke S., Dadap J.I. // J. Opt. Sos. Am. B. 2011. V. 28. N 6. P. 1374. doi 10.1103/PhysRevB.79.155420

[12] Капшай В.Н., Шамына А.А. // ОПт. и спектр. 2017. T. 123. № 3. C. 416-429. doi 10.7868/S003040341709015X; Kapshai V.N., Shamyna A.A. // Opt. Spectrosc. 2017. V. 123. N 3. P. 440. doi 10.1134/S0030400X17090144

[13] Шамына А.А., Капшай В.Н. // Опт. и спектр. 2018. Т. 124. № 1. C. 105-121. doi 10.21883/OS.2018.01.45366.176-17; Shamyna A.A., Kapshai V.N. // Opt. Spectrosc. 2018. V. 124. N 1. P. 103. doi 10.1134/S0030400X18010198

[14] Roke S., Bonn M., Petukhov A.V. // Phys. Rev. B. 2004. V. 70. N 11. P. 115106. doi 10.1103/PhysRevB.70.115106 